\title{
EVALUATION OF THE DISTAL RESECTION MARGIN VASCULARITY IN THE SUPERIOR RECTAL ARTERY PRESERVATION IN SIGMOIDECTOMY FOR SIGMOID COLON CANCER
}

\author{
By \\ Mohammed L. Abd El-Mawgod, Saeed H. El-Bendary and Mohammed \\ M. Ahmed \\ Department of General Surgery, Faculty of Medicine, Al-Azhar University \\ Corresponding Author: Mohammed Lotfy Abd El-Mawgod Sweafy, \\ E-mail: $\underline{\text { dr_mlotfy2017@gmail.com }}$
}

\begin{abstract}
Background: Colorectal surgery has gained wide acceptance, especially in the treatment of cancer colon. Several techniques and modifications have been proposed to improve morbidity and mortality associated with left hemicolectomy.

Objective: To evaluate the technique of superior rectal artery preservation in sigmoidectomy for sigmoid colon cancer, as regard impact on both radicality and vascularization in the remaining rectum.

Patients and Methods: A prospective study had been conducted on Bab El Sharia University Hospital and El-Sahel Teaching Hospital from March 2019 to March 2020. Thirty patients who are posted for sigmoidectomy with preservation of superior rectal artery had been included in the study.

Results: That mean of intraoperative blood loss was $35.64 \mathrm{ml}$ and $6.7 \%$ of patients have intraoperative bleeding. The mean of harvested lymph nodes was 19.27. One patient needed conversion to open surgery. As regard mean of operation time, it was $190.73 \mathrm{~min}$.

Conclusion: Superior rectal artery preservation sigmoidectomy for colon cancer was a beneficial technique guarding against post-operative anastomosing leakage, important oncologic principles as lymph node (LN) metastasis and recurrence should be taken in consideration should be kept in mind before performing that procedure.
\end{abstract}

Keywords: Sigmoid Cancer, Superior Rectal Artery, Sigmoidectomy, Colon Cancer.

\section{INTRODUCTION}

Colorectal surgery has gained wide acceptance, especially in the treatment of cancer colon. Several techniques and modifications have been proposed to improve morbidity and mortality associated with left hemicolectomy (Masoni et al., 2012).
Colorectal anastomotic dehiscence following these procedures significantly affects morbidity and mortality as well as treatment costs, especially in patients with benign conditions (Lehmann et al., 2011).

Ischemia or poor irrigation in the anastomosis is a major cause of dehiscence. Another complication worthy 
of mention, anterior rectal resection syndrome (ARRS) is characterized mainly by incontinence and/or fecal urgency, soiling, alternating bowel function and fragmented stools. Denervation of the distal colon segment is the main cause of ARRS following sigmoid resection (Dobrowolski et al., 2010).

The risk of dehiscence may be reduced by adopting a range of techniques (e.g. protective ostomy, mechanical stapling, double-layered suture, compression anastomosis clips, endo-luminal compression anastomosis rings and bioabsorbable Seam-guard ${ }^{\circledR}$ rings). One such technique, superior rectal artery (SRA) preservation, has been described for patients with proximal sigmoid and descending colon cancer with adequate lymphadenectomy (Sekimoto et al., 2011).

Although it is inadvisable in oncological surgery as it reduces chances of satisfactory lymphadenectomy. SRA preservation can reduce the risk of colon denervation, favouring postoperative bowel function and avoiding ARRS. The superior, middle and inferior rectum is irrigated mainly by the SRA. SRA preservation also preserves pelvic circulation and innervation, with reduced risk of functional impairment (sexual, urinary, evacuatory and fertility), but in addition to prolonging surgery, the procedure requires surgical skill and detailed knowledge of local anatomy to dissect the correct layer and ligate the sigmoid branches (Doryane et al., 2017).

During the classical radical treatment for sigmoid colon cancer, especially in patients with advanced diseases, ligation of the inferior mesenteric artery (IMA) at its root is typically performed to achieve better dissection of central and intermediate lymph node groups and improve the surgical outcomes. However, the blood supply to the left colon artery (LCA), sigmoid artery, and superior rectal artery (SRA) is blocked after the ligation at the root of the IMA. While the healing of the anastomosis is the most important factor affecting the success of the surgery, blood supply is one of the key factors affecting the anastomotic healing.

The blood supply to the proximal resection margin of the descending colon and the distal resection margin of the rectum tends to be poor in patient with abnormal vascular anatomy. Therefore, the operator has to mobilize the splenic flexure to search for a well-supplied segment of the intestine to establish anastomosis with the rectum, which often requires the mobilization of splenic flexure or even the removal of part of the descending colon (Shi et al., 2018).

We aimed in this study to evaluate the technique of superior rectal artery preservation in sigmoidectomy for sigmoid colon cancer, as regard impact on both radicality and vascularization in the remaining rectum.

\section{PATIENTS AND METHODS}

This prospective study had been conducted on Bab El Sharia University Hospital, El-Sahel Teaching Hospital from March 2019 to March 2020. Thirty patients who were posted for sigmoidectomy with preservation of superior rectal artery had been included in the study.

\section{Inclusion criteria:}

1. Patients aged up to 70 years. 
2. Not known inflammatory conditions.

3. Radical surgical intervention is the optimal line of treatment.

\section{Exclusion criteria:}

1. Patients on anticoagulant treatment with altered blood clotting or immune system.

2. Patients having distant metastasis especially in liver.

All patients were subjected to full history taking, examination and preoperative preparation.

\section{Surgical techniques:}

The operation started with an incision on the right surface of the sigmoid mesocolon. This was then elongated in the cephalad and caudal directions, expanding the dissection layer just beneath the mesorectal fascia and preserving the hypogastric nerve.

The incision in the serosa and the mobilization of the mesosigmoid continued to the root of the IMA. At this point, the lymph nodes and adipose tissue at the root of the IMA were dissected and the wall of the artery was exposed. Lymph nodes were then detached from the artery by peeling off the vascular sheath along the IMA and the SRA in order to expose the bifurcation of the Left colic artery and the sigmoid arteries. When the SRA was preserved, the arteries were not divided here. The sigmoid and descending mesocolon were mobilized in a medial to lateral approach to maintain the former dissecting layer to avoid injury to the gonadal vessels and the ureter, and then the lateral attachment of the sigmoid and descending colon was dissected.

In SRA-preserving sigmoidectomy, the precise location of the tumor and the distribution of the arteries were confirmed to determine which artery to divide. The arteries feeding the tumor were ligated and divided at the bifurcation from the SRA. The artery was then divided, and resections of the colon and end-to-end anastomosis were performed by hand sewing or circular stapler.

\section{Ethical consideration:}

The study was approved by the Ethics Board of Al-Azhar University and an informed written consent was taken from each participant in the study.

\section{Statistical analysis:}

The collected data organized, tabulated and statistically analyzed using statistical package for the social sciences (SPSS) version 22 (SPSS Inc, Chicago, USA). For qualitative data, frequency and percent distributions were calculated. For quantitative data, mean, standard deviation (SD), minimum and maximum were calculated. Statistical significance was defined as $\mathrm{P}$ value $<0.05$. 


\section{RESULTS}

This study was conducted on 30 patients with mean age of 63.42 years with male predominance about $60 \%$.

Regarding the medical history of patients, $46.7 \%$ have hypertension, $13.3 \%$ have D.M, $20 \%$ have hyperlipidemia and $10 \%$ have coronary heart disease. The tumor size mean was $43.81 \mathrm{~mm}$. The ASA score I was $33.3 \%$, score II was $60 \%$ and score III was $6.7 \%$. As regard to tumor depth, Tis was 3.3\%, T1 20\%, T2 13.3\%, T3 $50 \%$, T4a $10 \%$ and T4b was $3.4 \%$. $53.3 \%$ of patients have node involvement (Table 1).

Table (1): Medical history and Characters of colorectal tumor of studied patients

\begin{tabular}{|l|c|}
\hline Cases & No (\%) \\
\hline $\begin{array}{l}\text { Pedical history: } \\
\text { Hypertension No (\%) }\end{array}$ & $14(46.7 \%)$ \\
\hline Diabetes mellitus No (\%) & $4(13.3 \%)$ \\
\hline Hyperlipidemia No (\%) & $6(20 \%)$ \\
\hline Coronary heart disease No (\%) & $3(10 \%)$ \\
\hline Colorectal tumor: & \\
\hline ASA score No (\%) $_{\text {I }}$ & $10(33.3 \%)$ \\
II & $18(60 \%)$ \\
III & $2(6.7 \%)$ \\
\hline Tumor size (mm) Mean \pm SD & $43.81 \pm 7.24$ \\
\hline Tumor depth ${ }_{\text {No }(\%)}$ Tis & $1(3.3 \%)$ \\
T1 & $6(20 \%)$ \\
T2 & $4(13.3 \%)$ \\
T3 & $15(50 \%)$ \\
T4a & $3(10 \%)$ \\
T4b & $1(3.3)$ \\
N (-) & $14(46.7 \%)$ \\
N (+) & $16(53.3 \%)$ \\
\hline
\end{tabular}

That mean of intraoperative blood loss was $35.64 \mathrm{ml}$ and $6.7 \%$ of patients have intraoperative bleeding. The mean of harvested lymph nodes was 19.27. One patient needs conversion to open surgery. As regard mean of operation time, it was 190.73 min (Table 2). 
EVALUATION OF THE DISTAL RESECTION MARGIN VASCULARITY..

859

Table (2): Intraoperative data of studied patients

\begin{tabular}{|l|c|}
\hline Cases & No $(\%)$ \\
\hline Blood loss (mL) & $35.64 \pm 6.28$ \\
Mean \pm SD & $(10-245)$ \\
Range & $19.27 \pm 3.15$ \\
\hline Harvested lymph nodes & $3-34$ \\
Mean \pm SD & \\
Range & $190.73 \pm 24.41$ \\
Operation time (min) & $150-360$ \\
Mean $\pm S D$ & $2(6.7 \%)$ \\
Range & $1(3.3 \%)$ \\
\hline Intraoperative bleeding ${ }_{\text {No }(\%)}$ & \\
\hline Conversion to open surgery ${ }_{\text {No }}(\%)$ & \\
\hline
\end{tabular}

The postoperative morbidity was

patient needed reoperation. The mean of $13.3 \%$, mortality was $3.3 \%$, and one

hospital stay was 7.22 day (Table 3).

Table (3): Postoperative complications of studied patients

\begin{tabular}{|c|c|}
\hline $\begin{array}{ll}\text { Postoperative complications } & \text { Cases } \\
\end{array}$ & No $(\%)$ \\
\hline Postoperative morbidity № (\%) & $4(13.3 \%)$ \\
\hline Pneumonia No (\%) & $1(3.3 \%)$ \\
\hline Pulmonary embolism № (\%) & $0(0 \%)$ \\
\hline Bowel obstruction № (\%) & $2(6.7 \%)$ \\
\hline Anastomotic leakage № (\%) & $0(0 \%)$ \\
\hline Anastomotic bleeding o (\%) & $0(0 \%)$ \\
\hline Duodenal ulcer bleeding № (\%) & $0(0 \%)$ \\
\hline Wound infection No (\%) & $1(3.3 \%)$ \\
\hline Mortality № (\%) & $1(3.3 \%)$ \\
\hline Reoperation ${ }_{\text {No }}(\%)$ & $1(3.3 \%)$ \\
\hline Hospital stay (day) Mean \pm SD & $7.22 \pm 2.53$ \\
\hline
\end{tabular}

\section{DISCUSSION}

Colorectal cancer (CRC) is a major public health problem. The $3^{\text {rd }}$ most commonly diagnosed cancer in males and the $2^{\text {nd }}$ in females worldwide. It is the $4^{\text {th }}$ cause of cancer death globally and over $9 \%$ of all cancer incidences with an estimated 1.4 million cases annually (Singh et al., 2017).

As regard demographic data of studied patients, the mean of patient's age was $(63.42 \pm 11.35 \mathrm{SD})$ and ranged from 38 to 69 year and $60 \%$ were males while females were $40 \%$. The mean of BMI of studied patients was $23.61 \mathrm{~kg} / \mathrm{m}^{2}$.

Shi et al. (2018) evaluated the outcomes of Laparoscopic radical treatment with preservation of left colon artery and superior rectal artery for sigmoid colon cancer. The mean age of the studied cancer sigmoid patients was $65.9 \pm 12.05 \mathrm{SD}$ and ranged $39-88$ years. $55 \%$ of them were males and the rest $45 \%$ were females, which is near our results.

The mean tumor size was $43.81 \pm 7.24$ SD mm. The ASA score I was $33.3 \%$, 
score II was $60 \%$ and score III was $6.7 \%$. As regard to tumor depth, Tis was $3.3 \%$, T1 20\%, T2 13.3\%, T3 50\%, T4a 10\% and $\mathrm{T} 4 \mathrm{~b}$ was $3.4 \% .53 .3 \%$ of patients have node involvement.

As regarding operative data, the mean of intraoperative blood loss was (35.64 $\pm 6.28 \mathrm{SD}) \mathrm{ml}$ ranged $(10-245 \mathrm{ml})$ and $6.7 \%$ of patients have intraoperative bleeding. The mean of harvested lymph nodes was 19.27 ranged (3-34) LNS. One patient needs conversion to open surgery. As regard mean of operation time, it was $190.73 \mathrm{~min}$.

As regard postoperative complications of studied patients. Post-operative morbidity was $13.3 \%$, mortality was $3.3 \%$, and one patient needed reoperation. The mean of hospital stay was 7.22 day. Details of complications were as follow: Pneumonia 3.3\%, bowel obstruction $6.7 \%$, wound infection $3.3 \%$. No post-operative leakage was detected among our operated patients.

In the study of Wakahara et al. (2015), the overall postoperative morbidity was bowel obstruction, pneumonia, pulmonary embolism, and wound infection. No mortality or reoperation needed. Our study was better in morbidity and higher in mortality.

In the study of Ge et al. (2017) no serious complications related to the approach were encountered, anastomotic leakage never occurred in these patients without preoperative chemoradiation therapy, the patients had quick convalescence, as evaluated by the recovery of flatus passage $(2.8-1.5$ days), postoperative bed activity time (1.5 - 0.5 days), postoperative hospitalization (10.8 - 4.6 days), degree of postoperative pain for 48 hours $(2.5-0.5$, visual analog scale), drainage tube removal time (1.0 0.4 days).

Another study by Wakahara et al. (2014), they made comparison of laparoscopic sigmoidectomy with and without preservation of the superior rectal artery, and concluded that, Sigmoidectomy with SRA preservation can be performed without compromising the quality of lymph node dissection and relapse-free survival. No advantage of preserving the SRA could be demonstrated, but it is noteworthy that no anastomotic leakage was noted among the 57 patients in SRA preservation group and 1 leakage case observed in patients without preservation of the superior rectal artery group.

In the study of Singh et al. (2017) in their meta-analysis about long-term survival benefits of high and low ligation of inferior mesenteric artery in colorectal cancer surgery. They concluded that, the pooled data showed high ligation of IMA has a better survival benefit for the patients with IMA positive LNs. It signifies high ligation should be recommended for the advanced cases or with the suspected high risk of IMA lymphatic metastasis. The limited number of articles demands future high-powered, well-designed randomized controlled trials (RCTs) for the further reliable conclusion.

Regarding anastomosis leakage some studies have concluded, high ligation has no undisputable proof of increased survival. Although the usage of IMA high ligation plays an important role in the improvement of LN retrieval, the precision of tumor staging, and to avoid 
EVALUATION OF THE DISTAL RESECTION MARGIN VASCULARITY... 861

tension in low pelvic anastomoses (Guraya, 2016).

\section{CONCLUSION}

SRA preservation sigmoidectomy for colon cancer was a beneficial technique guarding against post-operative anastomosing leakage, important oncologic principles as L.N metastasis. Recurrence should be taken in consideration should be kept in mind before performing that procedure. We should pay attention for other factors that may contribute in leakage as adequate mobilization and nutritional state.

\section{REFERENCES}

1. Dobrowolski S, Hai S, Kobiela J and Sledzii Z. (2010): Should we preserve the inferior mesenteric artery during sigmoid colectomy? Neurogastroenterol Motil., 21(12): 1288-e123.

2. Doryane MRL, João PB, Gustavo $K$ and Univaldo ES. (2017): Importance of Superior Rectal Artery Preservation in Videolaparoscopic Rectosigmoidectomy 004 for Benign Disease. Adv Res Gastroentero Hepatol., 4(3): 58-61.

3. Ge L, Wang HJ, Wang QS, Zhao ZL and Lei C. (2017): The surgical technique of laparoscopic lymph node dissection around the inferior mesenteric artery with preservation of superior rectal artery and vein for treatment of the sigmoid and rectal cancer. Journal of Laparoendoscopic \& Advanced Surgical Techniques, 27(2):175-80.

4. Guraya SY. (2016): Optimum level of inferior mesenteric artery ligation for the left-sided colorectal cancer. Systematic review for high and low ligation continuum. Saudi Med J., $37: 731-6$.

5. Lehmann RK, Brounts LR, Johnson EK, Rizzo JA and Steele SR. (2011): Does sacrifice of the inferior mesenteric artery or superior rectal artery affect anastomotic leak following sigmoidectomy for diverticulitis? A retrospective review. Am J Surg., 201(5): 623627.

6. Masoni L, Mari FS, Nigri G, Favi F and Gasparrini M. (2012): Preservation of the inferior mesenteric artery via laparoscopic sigmoid colectomy performed for diverticular disease: real benefit or technical challenge: a randomized controlled clinical trial. Surg Endosc., 27(1): 199-6.

7. Sekimoto M, Takemasa I, Mizushima T, Ikeda $M$ and Yamamoto H. (2011): Laparoscopic lymph node dissection around the inferior mesenteric arterywith preservation of the left colic artery. Surg Endosc., 25(3): 861-866.

8. Shi D, Liang L, MaY, Li Q and Li X. (2018): Laparoscopic radical treatment with preservation of left colon artery and superior rectal artery for sigmoid colon cancer. Translational Cancer Research, 7(3):738-743.

9. Singh D, Luo J, Liu XT, Ma Z, Cheng $H$ and Yu Y. (2017): The long-term survival benefits of high and low ligation of inferior mesenteric artery in colorectal cancer surgery: a review and meta-analysis. Medicine, 96(47):124-9.

10. Wakahara T, Toyokawa A, Ashitani H, Tsuchida S and Hasegawa Y. (2015): Comparison of laparoscopic sigmoidectomy with and without preservation of the superior rectal artery: A single-institution retrospective study. Asian Journal of Endoscopic Surgery, 8(1):29-33.

11. Wakahara T, Toyokawa A, Awadu M, Takahashi T, Tsuchida $S$ and Hasegawa $Y$. (2014): Laparoscopic sigmoidectomy with preservation of the superior rectal artery: usefulness of bipolar scissors. Journal of Japanese College of Surgeons, 39(2):155-9. 


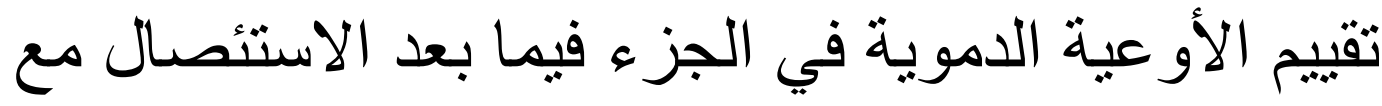

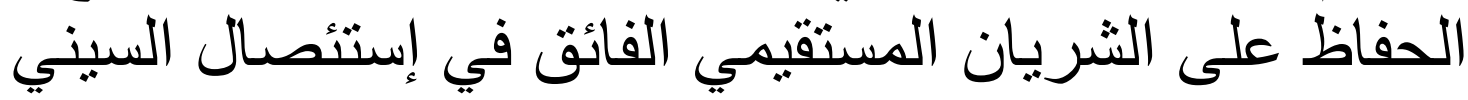
لسرطان القولون السيني الفي في الفي

محمد لطفي عبد الموجود سويفي, سعيد حسني البنداري, محمد ممدوح احمد قسم الجراحة العامة، كلية الطب، جامعة الأزهر

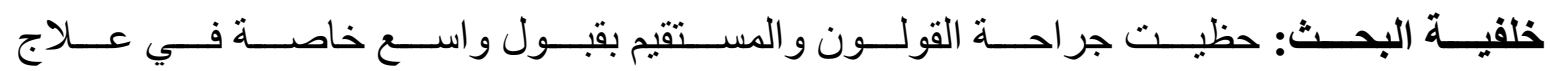

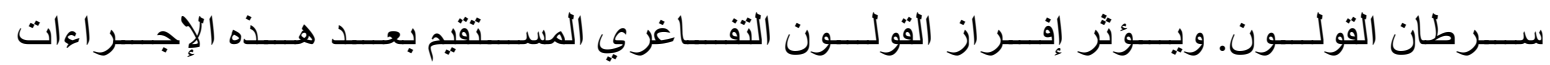

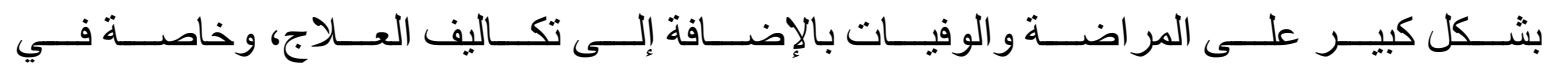
المرضى الذين يعانون من حالات حميدة.

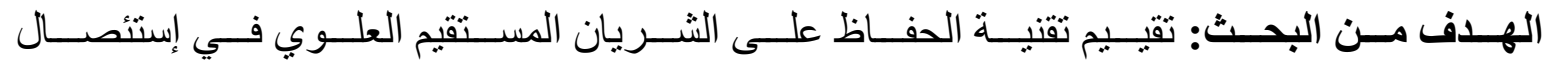

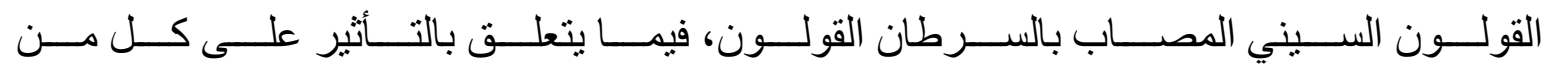
التطرف و الأو عية الدموية في المستقيم المتبقي.

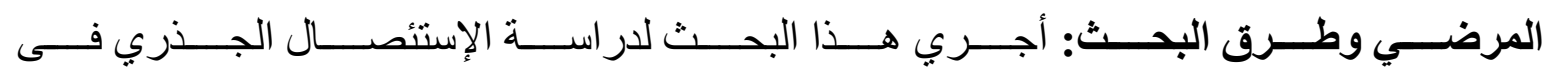

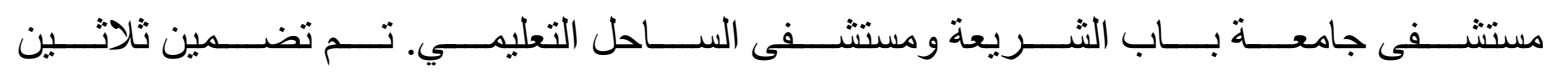

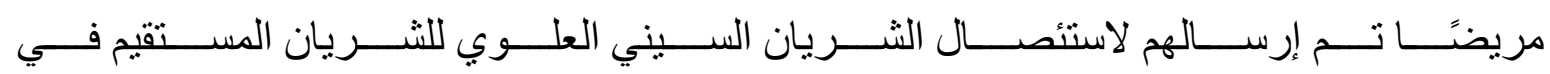

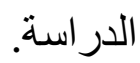

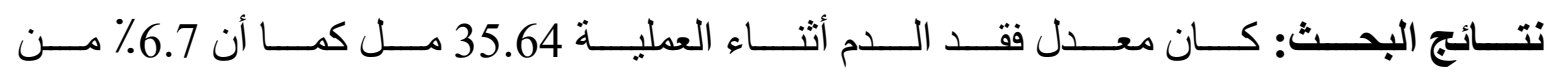

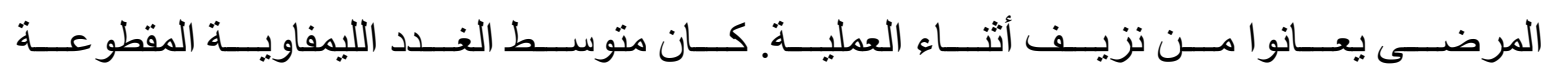

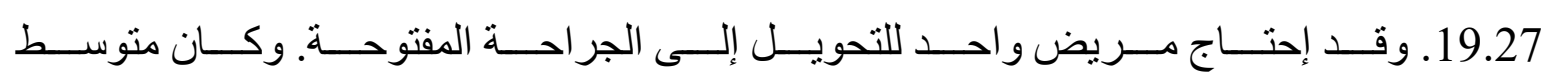

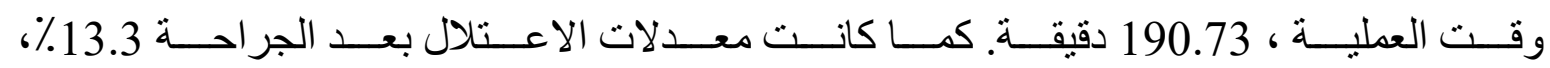

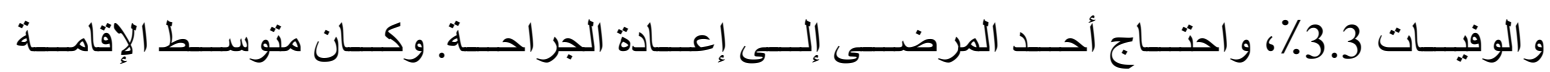
في المستشفى 7.22 يوماً.

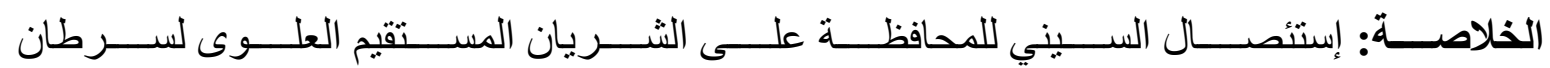

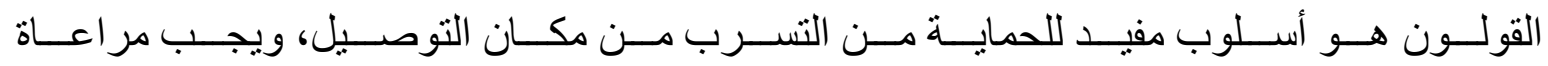

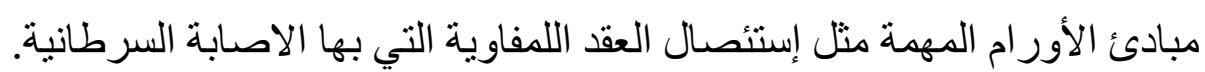

